\section{Epithelial ovarian cancer}

\author{
Alexander Reinthaller
}

Received: 5 April 2020 / Accepted: 7 April 2020

(c) Springer-Verlag GmbH Austria, part of Springer Nature 2020

Epithelial ovarian cancer is the leading cause of death among all gynecologic cancers in developed countries. The estimated number of newly diagnosed ovarian cancers per year in Europe is approximately 66,000 with 43,000 deaths. One of the main factors contributing to the high death-to-incidence rate is the advanced stage of the disease defined by the spread of the disease outside the pelvis at the time of the initial diagnosis. More than two thirds of patients are diagnosed at an advanced stage of the disease. Late-stage presentation has a 5-year survival rate between 30 and $50 \%$ depending on the expertise of the treating center. In contrast early stage disease can be cured in $70-90 \%$ of individuals. The main reason for the advanced stage presentation is the lack of early symptoms during the development of epithelial ovarian cancer.

This issue will cover the latest aspects of screening, identifying high-risk populations and the options to improve early diagnosis. New developments for early diagnosis and prevention that leverage molecular genomics are under investigation.

Radical surgery is still a corner stone in the treatment of advanced epithelial ovarian cancer. There have been major efforts in the last few years to elucidate the impact of radical surgery on progressionfree and overall survival in well-designed randomized controlled trials. The results of these trials and their impact on clinical practice will be revisited.

In recent years there have been major improvements in the understanding of the biology of invasive

Prof. A. Reinthaller, M.D. (ه)

Department of Gynecology and Gynecologic Oncology, Gynecologic Cancer Unit, Comprehensive Cancer Center, Medical University of Vienna, Waehringer Guertel 18-20, 1090 Vienna, Austria

alexander.reinthaller@meduniwien.ac.at epithelial ovarian cancer and this knowledge has led to changes in clinical practice.

Genomic predisposition to high grade ovarian cancer is recognized in up to $20 \%$ of affected women. Breast cancer susceptibility genes BRCA1 and BRCA2 have been identified as causative genes involved in the origin of hereditary ovarian cancer. Deleterious mutations (germline and somatic) in the BRCA genes and other double-strand DNA break repair genes causing a defect in DNA repair called homologous repair deficiency (HRD) are frequently associated with high grade serous epithelial ovarian cancers. Other mutations leading to mismatch repair deficiencies and micro satellite instability as present in patients with Lynch syndrome can lead to high grade endometrioid and clear cell cancers. In patients with HRD poly(adenosine diphosphate[ADP]-ribose) polymerase inhibitors (PARPI) showed impressive effectiveness in the treatment of platinum-sensitive recurrent ovarian cancer and have now moved into first-line therapy. Clinical trials investigating the combination of PARPI with other active agents like antiangiogenic agents or checkpoint inhibitors have been already completed or are currently recruiting patients. Data, results, and possible future implications about PARPI will be covered in this issue.

The introduction of checkpoint inhibitors (CI) into the therapy of a variety of different tumors led to a new understanding of immuno-oncology in the treatment of cancer. High grade serous ovarian cancer turned out to be an immunologically cold tumor with lowto-moderate response to CIs. The combination of CIs with chemotherapy or other targeted therapies might improve efficacy. Other types of ovarian cancer like high grade endometrioid tumors seem to be more susceptible to CIs. The possible role of CIs in the treatment of epithelial ovarian cancer will be discussed. 


\section{editorial}

Conflict of interest A. Reinthaller declares that he has no competing interests.

\section{References}

\section{Further Reading}

1. Aust S, Seebacher-Shariat V. Screening for ovarian cancer: is there still hope? memo. 2020. https://doi.org/10.1007/ s12254-019-00563-2.

2. Schwameis R, Paspalj V, Kranawetter M, Polterauer S. Ovarian cancer surgery. Game changing innovations. memo. 2020. https://doi.org/10.1007/s12254-020-00596-y.

3. Zeimet AG, Wieser V, Knoll K, et al. PARP inhibitors in the treatment of ovarian cancer. memo. 2020. https://doi.org/ 10.1007/s12254-020-00585-1.
4. Bartl Th, Paspalj V, Polterauer S, Grimm C. Current state and perspectives of checkpoint inhibitors in ovarian cancer treatment. memo. 2020. https://doi.org/10.1007/s12254020-00579-z.

Publisher's Note Springer Nature remains neutral with regard to jurisdictional claims in published maps and institutional affiliations.

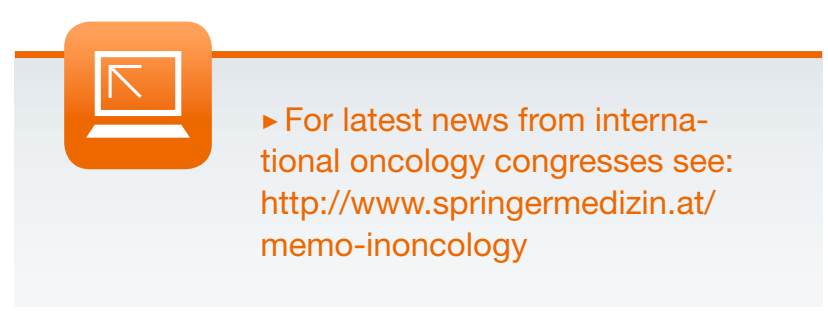

